\title{
Zur historischen Phonetik und Phonologie des Umlauts im Deutschen'
}

\author{
Gregory $\mathrm{K}$. Iverson \\ University of Wisconsin-Milwaukee
}

\author{
Joseph C. Salmons \\ University of Wisconsin-Madison
}

Seit mehr als 60 Jahren dominiert in der historisch-phonologischen Umlaut-Landschaft EIN Aufsatz, eine vier-seitige Skizze des althochdeutschen Umlauts von W. Freeman Twaddell. Keller (1978:160) nennt diese Theorie 'one of the finest achievements of American linguists'. Ähnliche Lobsprüche findet man mehrmals in der Literatur und der Artikel bleibt bis heute noch DER Eckpfeiler der Umlaut-Debatte (s. Krygier 1997, Schulte 1998).

In den letzten paar Jahren haben wir mit einigen Kollegen- Anthony Buccini, Garry Davis, David Fertig, Dave Holsinger, Robert Howell, Regina Smith —einen neuen Ansatz entwickelt, die wir "ingenerate Umlaut" nennen. "Ingenerate" heißt hier ungefähr 'vorprogrammiert, inhärent, angeboren' und deutet darauf hin, daß wir die Wurzeln vom Umlaut in der Phonetik - noch genauer: in der Koartikulation - suchen. Auch meinen wir, die allmähliche Entfaltung des Prozesses in den "Ausnahmen" zum Umlaut sehen zu können, mit anderen Worten genau in den umlautlosen Formen, die in der Twaddellschen Tradition als willkürliche Ergebnisse der Analogie gesehen werden müssen.

\section{Ein Vergleich: Zwei Ansätze}

Twaddell beschäftigt sich in seinem Aufsatz vom Jahre 1938 spezifisch mit der relativ frühen Schreibung, der schon um 750 u.Z. auftaucht, vom sogenannten "Primärumlaut", oder der partiellen Assimilation von $/ \mathrm{a} / \mathrm{zu} / \mathrm{e} /$ vor einem $/ \mathrm{i}, \mathrm{j} /$ einer folgenden unbetonten Silbe im Kontrast zu der sehr späten Schreibung von allen anderen Umlauterscheinungen, die erst Jhh. nach der Abschwächung und endlich dem Verlust von den alten, umlautwirkenden Endvokalen regelmäßig vorkommt.

Für Twaddell ist entscheidend, daß alle Umlauterscheinungen gleichzeitig passiert sind, ob Primärumlaut (1a), Umlaut in der Umgebung von der sogenannten Hinderung oder Hemmung (1b), "Sekondärumlaut" von Hinderungsumgebungen und von langem /a/ (1c) oder sonstige Phänomena (1d). Twaddell macht zum ersten Mal die Bedeutung der Phonologisierung in der Evolution von Umlaut deutlich und verwirft damit die bisherigen Umlautheorien.

Leider fängt mit dem Artikelchen eine Tradition an, die bis heute in der Umlautliteratur überall zu spüren ist, wie wir gleich sehen werden: Die westgermanischen Umlautfakten werden drastisch vereinfacht und besonders die umlautlosen Formen werden völlig ignoriert.

(1) a. Primärumlaut, ahd

$\begin{array}{ll}\text { gast } \sim \text { gesti } & \text { 'Gast, Gäste' } \\ \text { lamb } \sim \text { lembir } & \text { 'Lamm, Lämmer' } \\ \text { fasto } \sim \text { festi } & \text { 'fest', adv. und adj. }\end{array}$

\footnotetext{
${ }^{1}$ Der vorliegende Aufsatz wurde am 5. Mai 1999 bei ZAS vorgetragen. Er basiert auf einer englischen Version, die demnächst als Iverson \& Salmons (1999) erscheint.
} 
b. Hinderung, ahd. (aber mhd. 'Sekundärumlaut') $\begin{array}{ll}\text { maht } \sim \text { mahti } & \text { 'Macht, Mächte' (auch dial. mehti) } \\ \text { haltan } \sim \text { haltis } & \text { 'halten, hält' (dial. heltis) } \\ \text { starch } \sim \text { starchiro } & \text { 'stark, stärker' (auch sterchiro) }\end{array}$

c. Sekundärumlaut von $/ \mathrm{a} /$ und $/ \mathrm{a}: /$, mhd. värwen, verwen 'färben, Farbe', cf. ahd. farawen zähere 'Tränen', cf. ahd. zahari

d. Allgemeiner Umlaut von allen [+hinten] Vokalen vor /i.j/ ahd nhd gruoni grün 'green' skoni schön 'beautiful'

Mit unserer Alternative versuchen wir, alle Umlautdaten nicht nur phonologisch adäquat zu erklären, sondern auch phonetisch und historisch. Die historischen Anfänge sehen wir im Primärumlaut. Wie wir meinen, hat sich der Umlaut von diesem Kern verbreitet und verallgemeinert.

Im Mittelpunkt steht für uns eine Reihe von Unterschieden zwischen Primärumlaut und NichtPrimärumlaut, also zwischen den Daten in la und denen in lb-d. Diese Unterschiede, in (2) zu lesen nach Iverson \& Salmons 1996, führen wir darauf zurück, daß Primärumlaut chronologisch früher passiert ist, während die Stammvokale in den Typen 1b-d später umgelautet wurden, als der ursprüngliche Umlautprozeß systematisch ausgebaut und verallgemeinert wurde.

\begin{tabular}{ll} 
PRIMÄRUMLAUT & NICHT-PRIMÄRUMLAUT (SEKONDÄR uSW) \\
\hline genaue phonetische Umgebung & allgemeiner Prozeß (+hint $\rightarrow$-hint__i,j \\
"Structure-Preserving" & schafft neue Segmente \\
konsequent durchgeführt & unvollkommen (Ausnahmen im Obdt.) \\
sehr früh geschrieben & Jhh. später geschrieben
\end{tabular}

Primärumlaut betrifft nur einen kurzen Vokal, /a/, und auch dann nicht, wenn in manchen Dialekten $/ \mathrm{rC} /, / \mathrm{Cl} / \mathrm{hC} / \mathrm{zwischen} / \mathrm{i}, \mathrm{j} /$ und $/ \mathrm{a} /$ stehen. Nicht-Primärumlaut betrifft dagegen alle hintere Vokale vor $/ \mathrm{i}, \mathrm{j} /$, egal welche Konsonantenverbindungen dazwischenstehen. Primärumlaut ist Structure-Preserving im Sinne Kiparsky, während Nicht-Primärumlaut NUR neue Segmente. schafft. Primärumlaut wird im ganzen Kontinental-Westgermanischen streng konsequent durchgeführt - abgesehen von der Variation in der Schreibung, u.ä. - - während NichtPrimärumlaut in manchen Gegenden gar nicht $z u$ finden ist (in westniederländischen Dialekten z.B.) und deutliche Unregelmäßigkeiten in anderen (im Oberdeutschen) aufweist.

Ingenerate Umlaut bringt im Vergleich zu Twaddellschen und Neo-Twaddellschen Umlauttheorien erkennbare deskriptive, empirische und theoretische Vorteile mit sich.

Zum ersten: Deskriptive Probleme entstehen für die traditionelle Ansicht, weil eine einzige, einheitliche Beschreibung von den Daten in 1a und 1b unmöglich ist (Iverson \& Salmons 1996:78, Howell \& Salmons 1997).

Primärumlaut wird gerade in der Umgebung von $1 \mathrm{~b}$ verhindert, aber anderseits gibt es im Mhd. einen Umlaut gerade in dieser Umgebung. Ohne die Hinderungsdaten in $1 \mathrm{~b}$, würde eine lineare Regel wie unter (3) genügen. Solche Regeln findet man oft genug in der frühen generativen Literatur (King, Kiparsky, u.a.). 
(3) Einheitsregel für Umlaut (typisch für frühe generative Forschung)

$\left[\begin{array}{c}\mathrm{V} \\ (\text {-lang) }\end{array}\right] \rightarrow\left[\begin{array}{c}- \text { hint } \\ \text {-tief }\end{array}\right] / \mathrm{C}_{\Omega}\left[\begin{array}{l}-\mathrm{knns} \\ - \text { hint } \\ + \text { hoch }\end{array}\right]$

Kurzes /a/ wird normalerweise im Althochdeutschen umgelautet. Dieser Umlaut findet aber nicht in der Umgebung von $/ \mathrm{xC} /, / \mathrm{LC} /$ im Althochdeutschen statt, sondern erst im Mittelhochdeutschen. Dieser Unterschied hieße, daß die Teile von der Regel in Klammern - (-lang) — nur dann ungültig wäre, nur wenn die hindernden Konsonantenverbindungen zwischen $/ \mathrm{a} / \mathrm{und} / \mathrm{i} / \mathrm{stehen}$. Mit anderen Worten müßte man, um (3) deskriptiv adäquat zu machen, die offenbar unnatürliche und sicherlich unerlernbare Bedingung unter (4) hinzufügen:

(4) (-lang) hindert Umlaut, aber nur wenn $\mathrm{C}_{\mathrm{o}}$ enthält $/ \mathrm{xC} /, / \mathrm{LC}$, etc.

Dieser Fehler gehört keineswegs ausschließlich zu den Generativisten; er setzt ja einfach die Analyse der Strukturalisten sehr unkritisch fort. Und wir sind nicht die ersten, die den Fehler erkannt haben: Charles Russ (1977:219) meinte schon vor mehr als 20 Jahren in einem Aufsatz über die moderne Geschichte der Umlautforschung: "die Wirkung der umlauthemmenden Konsonantenverbindungen wird nicht berücksichtigt". Soweit wir wissen hat bis jetzt aber niemand die theoretischen Konsequenzen aus diesem Fehler gezogen, was wir nun tun wollen.

Auf der empirischen Ebene ermöglicht uns die neue Theorie, fast alle Umlautdaten im Rahmen von einer einzigen Analyse zu erfassen. Diejenigen, die Twaddells Skizze zu einer allgemeinen Theorie des Umlauts entwickelten - hier denkt man vor allem an Penzl und Antonsen -, haben eingesehen, daß alle unumgelauteten Vokale, die ursprünglich vor $\mathrm{i} / \mathrm{j}$ standen, analogische Rückumlautformen sein müssen. In der generativen Tradition hat man bewußt oder unbewußt die umlautlosen Formen völlig außer acht gelassen. Wir suchen aber phonetische, phonologische und diachrone Erklärungen auch für diese Daten, in erster Linie für die schon erwähnten Ausnahmen zum Primärumlaut ( $1 \mathrm{~b}$ ), für die umlautlosen Formen in den modernen oberdeutschen Dialekten, in (6), sowie auch für Umlaut im Niederländischen.

Zum ersten Fall bieten wir eine einheitliche phonologische Analyse von den primärumlauthemmenden Konsonantenverbindungen, je nach althochdeutschem Dialekt (siehe (5.2) nach Iverson, Davis \& Salmons 1994). Wir behaupten, daß Vokalisierung von KodaLiquiden sowie Schwächung von Koda / $\mathrm{x} /$ Primärumlaut hindert. Wir folgen hier Howells Analyse der altenglischen Brechung - 5.1. Wo frühere Spezialisten angenommen haben, daß Brechung ein Ergebnis von hinteren Varianten der Konsonanten /, $\mathrm{r}, \mathrm{x} /$ war, zeigt Howell, daß man brechungähnliche Vokale in modernen deutschen, niederländischen und englischen Dialekten dort und nur dort findet, wo $/, \mathrm{r}, \mathrm{x} /$ in Kodastellung reduziert bzw. vokalisiert worden sind.

Für Umlaut sieht es ähnlich aus, aber die Parallelen sind noch genauer: Primärumlaut wird nur in den althochdeutschen Dialekten gehemmt, wo die modernen Dialekte Vokalisierung von den hemmenden Koda-Konsonanten aufweisen. Im Bairischen werden z.B. Koda 1 und r vokalisiert und altbairische Texte zeigen Hemmung vor $l$ und $r$ plus Obstruent. Im Alemannischen ist die moderne vokalisierte Form von Koda / $\mathrm{r} /$ weniger üblich, und die Verbindung $\mathrm{rC}$ hindert in den ältesten alemannischen Texten weniger konsequent als $1 \mathrm{C}$. Vergleiche 5.2 und 5.3-6. Wie schon oben erwähnt, betrifft Primärumlaut nur kurzes /a/, und die Vokalisierung von Koda-Liquiden heißt natürlich, daß der Silbenkern oder Nukleus nicht mehr den Bedingungen von Primärumlaut entsprichttrifft. 
(5.1) "Brechung" im Altenglischen (Howell 1991)

$\mid \mathrm{e} / \rightarrow\langle\mathrm{ea}\rangle,\langle\mathrm{eo}\rangle$ vor $\langle\mathrm{lC}\rangle,\langle\mathrm{rC}\rangle,\langle\mathrm{hC}\rangle$
a. ae. we orban 'werden' vs. as. werdan
b. ae. eald 'alt' vs. as. ald
c. ae. bearn 'Kind' vs. as. barn

(5.2) Umlauthinderung, ahd $(\mathrm{Fr}=$ Fränkisch, Bair = Bairisch, $\mathrm{Al}=$ Alemannisch $)$

a. <ht>, <hs>: hindert auf dem ganzen ahd Gebiet: Fr, Bair, Al (außer Otfrid): maht/mahti; mahtig 'Macht' nom.sg./pl.; adj. naht/nahti 'Nacht' nom.sg./pl.

b. <hh>, <ch>: hindert im Bair und Al, nicht im Fr (z.B. Tatian): sachan/sahhis/sachit 'sehen' 1./2./3. sg.(T forsehhis/-sehhit) gimachida 'Gemeinsamkeit'

c. $/ \mathrm{CC} /, / \mathrm{rCl}$ : hindert sporadisch im $\mathrm{Fr} ; / \mathrm{C} / \mathrm{konsequent} \mathrm{im} \mathrm{Bair} \mathrm{und} \mathrm{Al} ; / \mathrm{rC} /$ üblich im Bair, gelegentlich im Al: haltanhaltis/haltit 'halten 1./2./3. sg. (Fr. heltis/heltit) waltan/waltit (Fr. weltit) 'walten, etc.' inf./3. sg.pres.

(5.3) Koda -1 Vokalisierung

Bair: Vokalisierung von $/ \mathrm{l} /$ : Hoiz 'Holz', moi 'Mal'

mhd fälke-lîn 'Falke' (Verkleinerungsform) = Mittelbair/Österr. fäụkl

Al: 'Wald' = waud Vorarlberg (Bregenzerwald)

(5.4) Koda $-\mathrm{r}$ Vokalisierung

Im Bair. weit verbreitet; ausgesprochen NICHT typisch für Al (s. Howell 1991).

(5.5) Reduktion von Koda $<\mathrm{h}\rangle(* / \mathrm{x} /)$

a. Häufig aber variabel in unbetonten Silben:

Bair und $\mathrm{Al}:<$ duruh $>$ (/durux/) $\rightarrow$ dur 'durch', weliher (/welixer/), welher (/welxer/) $\rightarrow$ weler 'welcher'; mhd niet $\sim$ niut vs. niht 'nicht', Formen mit/ohne $<\mathrm{h}>$.

b. Verlust sogar in betonten Silben:

retlîhe für rehtlîhe 'gerecht' (Otfrid)

Ripuarisch, andere fränkische Dialekte: brāt, braut für brachte, nātt, nēt, nęit, naut für

Nacht 'night', māt für machte, flępto für flechten

(5.6) Hyperkorrektion

heber $=$ eber 'Eber' (Monseer Fragmente)

hërda $=\ddot{e} r d a$ 'Erde' (Notker)

herbarmida = erbarmida 'Barmherzigkeit' (Fr.) [Hinderung + hyperkorr. h!]

Sogar mit Hinblick auf die berüchtigte oberdeutsche Umlautfeindlichkeit (siehe (6)), zeigen Howell \& Salmons (1997), daß ein phonetisch-phonologisches Prinzip im Spiel ist, nicht ad hoc, willkürliche Analogien. Wie wir zu behaupten wagen, setzt Assimilation voraus, daß zwei Segmente anders sind. Howell \& Salmons behaupten einfach, daß Umlaut zuerst Vokalpaare betrifft, die unterschiedlicher sind, während Vokale, die ähnlicher sind, erst später getroffen werden. Beim i-Umlaut wird also /a/ zuerst assimiliert, dann /o/ und endlich / $\mathrm{d} /$. Man erwartet also Ausnahmen gerade bei $/ \mathrm{u} /$. Das Prinzip gilt auch, mutatis mutandis, für a- und $u-U m l a u t$.

Der Artikulationsort von Konsonanten zwischen /i/ und /a/ spielt eine weiter komplizierende Rolle, besonders wo diese Konsonanten Geminaten sind. Koronale Obstruenten hindern Umlaut selten, labiale öfter und velare regelmäßig.

(6) Oberdeutsche Umlautlosigkeit (Schirmunski 1962:201-203)

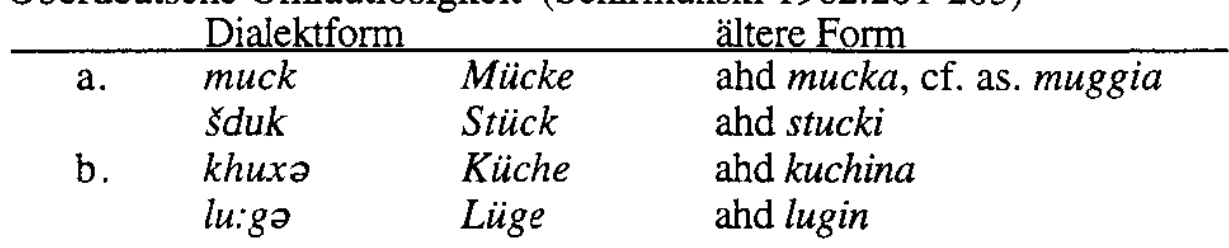


Die meisten oberdeutschen Formen ohne Umlaut sind, wie die Beispiele in (6), Wörter mit / $\mathrm{u} /$ als Stammvokal und velaren Obstruenten vor dem umlautauslösenden i oder j. Kurz gesagt: diese Daten spiegeln kein analogisches Chaos wieder, und sie brauchen nicht mehr verheimlicht oder vergessen zu werden. Ganz im Gegenteil sehen wir in diesen Daten den natürlichen evolutionären Weg von Umlaut im Deutschen und meinen, daß ohne sie keine vernünftige Erklärung von Umlaut möglich ist.

Der neue Ansatz genieBt noch andere empirische Vorteile im weiteren westgermanischen Raum. Im Niederländischen existiert eine Art Umlaut-Kontinuum: von den westlichen Dialekten, wo nur Primärumlaut stattgefunden hat, nach dem Osten und Süden, wo man schrittweise immer mehr Umlaut findet. Solche Daten lassen sich gar nicht mit den Auf-einen-Schlag-Umlauttheorien in Einklang bringen; sie passen aber ganz natürlich in die graduelle, phonetisch-phonologische Entfaltung von Umlaut, wie wir sie verstehen.

Theoretisch liegt es auf der Hand, daß sich Fernassimilationen seit der autosegmentalen Phonologie wesentlich leichter erklären lassen. Aber abgesehen von solchen Fortschritten, kommt "ingenerate Umlaut" dem alten theoretischen Grundprinzip der Ausnahmslosigkeit der Lautgesetze viel näher. Twaddells Folger mußten schon früh jeglichen Anspruch auf Regelmäßigkeit preisgeben, da ein gewisser Anteil der gesamten Daten nur analogisch zu verstehen war, einschließlich alle umlauthemmenden Konsonantenverbindungen, die vielen 'umlautfeindlichen' Formen des Oberdeutschen sowie die Dialektgeographie des niederländischen Umlauts ... alles in allem, eine erhebliche Menge von Problemfällen. Uns scheint es nach den Grundprinzipien der Wissenschaft klar genug, daß eine regelmäßige, phonetisch motivierte Erklärung eines Lautwandels einer ad hoc analogischen vorzuziehen ist.

Sodann widerspricht die Theorie von Umlaut als Monolith der bekannten Tendenz, daß sich komplexe Lautveränderungen aus relativ einfacheren, phonetisch motivierten entwickeln, wie Ohala u.v.a. seit Jahrzehnten behauptet haben.

Neulich schrieb Michael Schulte (1998:262) folgendes zur Umlautforschung im Nordischen:

Mit der Forderung nach einheitlicheren und eindeutigeren Erklärungen stellt sich zugleich die Frage nach einem exakteren Paradigma. ...

Wie sich zeigte, können die wirklich drängenden Fragen nicht durch die Anpassung oder Präzisierung alter Systeme, sondern nur durch die Artikulierung eines neuen Paradigmas gelöst werden. Gefordert ist ein in sich geschlossener Neuansatz, der nicht nur gewisse Detailprobleme, sondern den ganzen Problemkreis anvisiert.

Das stimmt natürlich auch für Umlaut im Westgermanischen, ja im Germanischen überhaupt. Aus diesem Vergleich von Twaddells Theorie des Umlauts und der Theorie des 'ingenerate' Umlauts schließen wir, daß die letztere konsequent aus deskriptiven, empirischen und theoretischen Gründen besser ist. Es ist Zeit, neo- und post-Twaddellsche Ansätze endlich loszuwerden. "Der in sich geschlossene Neuansatz" beginnt für uns mit dem phonetischen, koartikulatorischen Fundament des Umlauts.

\section{Die phonetische Basis vom “ingeneraten" Umlaut}

Wenden wir uns also jetzt der phonetischen Basis von Umlaut zu. Koartikulation in den modernen germanischen Sprachen erweist sich als stärker in den Umgebungen, wo historischer Umlaut stärker ausgeprägt war und schwächer, wo Umlaut unvollkommen geblieben ist. Diese Beziehung zwischen heutigen koartikulatorischen Erscheinungen und Umlaut ist aber nicht rein typologisch; wir sehen darin eine direkt kausale Beziehung: Koartikulation als phonetischer Prozeß führt zu Assimilationen, die später als Lautwandel sozusagen "versteinert" werden, aber so daß man noch die koartikulatorischen Fußabdrücke hier und dort noch deutlich sehen kann. 
Farnetani (1997:383-84) faßt das Verhältnis zwischen Koartikulation und Assimilation so zusammen: Coarticulation "... is a continuous motor process, increasing in magnitude in connected speech...", while assimilation "...is a categorical change, a language-specific grammatical rule. Assimilation is a consequence of coarticulation, an adaptation of language to speech constraints." Wir möchen hier betonen, daß genau wie Assimilation eine Folge der Koartikulation ist, so ist'auch Umlaut qua Lautwandel eine Folge von Assimilation.

Die bahnbrechenden Untersuchungen von Öhman (1966) und Keating (1988) und neuere Werke bis zur Gegenwart machen die Omnipräsenz von V-V Koartikulation in den Sprachen der Welt deutlich. Es scheint, daß i-Umlaut solcher subphonemischen Erscheinungen entsprungen ist. Wir vermuten, daß solche Koartikulation sehr früh vorhanden war und in gewissem Sinne die Entfaltung von Umlaut vorprogrammiert hat. Umlaut als synchroner phonologischer Prozeß entstand als Übertreibung von diesen Koartikulationen.

In den heutigen westgermanischen Sprachen führt die gleiche Art von Koartikulation sogar immer wieder zu umlautähnlichen Assimilationen. Unter (7) nennen wir ein paar solche Fälle, darunter das berühmte Beispiel der "Canadian Raising", einer Diphthongzentralisierung, die jetzt DAS typische Merkmal von einem kanadischen Akzent geworden ist. In beiden Fällen - Primärumlaut und Diphthongzentralisierung - erleichtem sich Sprecher innerhalb von Millisekunden den schweren Übergang von der hintersten, tiefsten Ecke des Vokalraumes hin zur vordersten, höchsten Ecke.

(7) "Canadian raising": /aj/, /aw/ $/ \mathrm{\Lambda j} /, / \Lambda \mathrm{w} /$ (bes. wo $/ \mathrm{a} /$ phonetisch kurz ist, wie $\mathrm{z.B}$. vor fortis/stimmlosen Konsonanten)

Siehe auch die Dialekte von Martha's Vineyard (New England), Outer Banks (North Carolina), "English Fens" (s. Britain 1997), etc.

Dieser assimilatorische Anfang hat im frühen Germanischen eine Parallele in der

Monophthongierung von alten *ai und *au. Braune/Eggers (1989) nach wird *ai zu /e:/ früher als *au zu /o:/ monophthongiert. Wir erklären diesen Unterschied einfach dadurch. daß die artikulatorische Entfernung von $/ \mathrm{a} / \mathrm{zu} / \mathrm{i}, \mathrm{j} /$ größer ist, als die von $/ \mathrm{a} / \mathrm{zu} / \mathrm{u} /$. Auch hier haben wir Ähnlichkeiten in den modernen germanischen Sprachen: In seiner Untersuchung von vokalischem Lautwandel in Martha's Vineyard kommt Labov zu dem Schluß daß: "... a rising first element of (ay) carries the first element of (aw) with it."

Monophthongierung von einem /ai/ Diphthong ist ein natürlicher Ausgangspunkt für Umlaut, weil die beiden Vokale, bzw. Vokal plus Halbvokal, phonetisch unmittelbar nebeneinander stehen. Aber noch auffälliger ist das Verhalten von /a/ und / $\mathrm{V}$, wo Obstruenten dazwischenstehen: Butcher \& Weiher (1976) untersuchten Koartikulation von [i, a, u] Paaren, die durch die Konsonanten [p, $\mathrm{t}, \mathrm{k}]$ unterbrochen wurden. Das Hauptergebnis der Studie ist: "A coarticulatory hierarchy among the vowels investigated such that [i] exerts the greatest coarticulatory influence and [a] the least" (1976:59). Ähnliche Ergebnisse findet man bei Magen (1989) für das Amerikanische, wo im Extremfall die Wirkungen von /i/ganze zwei Silben früher in einem Nonsenswort, [babəbi], instrumental meßbar sind.

In den modernen germanischen Sprachen sieht man Koartikulationen, die genau zur Entfaltung von Umlaut passen. Weiter bemerkenswert ist die Tatsache, daß es aus den phonetischen Vorgängen immer wieder zu umlautähnlichen phonologischen Assimilationen gekommen ist. Wir können . natürlich nicht vorhersagen, daß es eine zweite Umlautrunde im Germanischen geben wird, aber zumindest historisch sind die Würfel gefallen. 


\section{Umlauthinderung als Scheitern von Koartikulation}

Wie schon gesagt, Umlaut wurde durch gewisse Konsonantenverbindungen gehemmt, wenn sie zwischen $/ \mathrm{i} /$ und $/ \mathrm{a} /$ standen und Spuren einer Hemmung sieht man in oberdeutschen Dialekten, besonders bei Velarobstruenten. Wenn unsere These der subphonemischen Koartikulation als phonetische Quelle von allen Umlauterscheinungen richtig ist, dann sollte Koartikulation durch solche Konsonanten in den modernen Sprachen gehindert bzw. verhindert werden.

Glücklicherweise findet man Hinderung von Koartikulation über genau die richtigen Konsonanten: Konsonanten sind normalerweise in der V-V Koartikulation 'durchsichtig' oder 'transparent', aber Keating (1988) zeigt, daß dieser Prozeß durch Konsonanten gehemmt wird, wo diese Konsonanten für die Merkmale spezifiziert sind, die sonst angeglichen werden.

Die schon erwähnte Untersuchung von Butcher \& Weiher (1976) zeigt, daß koartikulatorische Wirkungen über alveolare Verschlußlaute relativ stark sind, während Koartikulation über velare Verschlußlaute bedeutend weniger sind. Ihre Erklärung:

It is noticeable that coarticulation extends across velar stops only when [i] (usually the most powerful coarticulator) influences [a] (always the weakest).... It appears that velar stops offer a much greater barrier to across-closure coarticulation than do alveolars.... This difference, naturally, makes good physiological sense when one considers that the alveolar articulation is made with the relatively independent tongue tip, leaving the body of the tongue free to continue the vocalic movement. Velar closures, on the other hand, involve far more of the main body of the tongue and are thus more likely to prevent the continuation of movements related to the vocalic gesture across the entire closure period (1976:71-72).

Leider gibt es soweit wir wissen keine Untersuchungen speziell über Geminaten, aber zumindest passen die Ergebnisse über Ort der Konsonantenartikulation in der V-V Assimilation sonst sehr gut zu den Fakten der oberdeutschen Umlautfeindlichkeit. Solche Experimente unterstützen natürlich auch unser Bild von einer differenzierteren Entfaltung von Umlaut.

Die phonetische Literatur zur Koartikulation bietet also weitere Stützen für unsere These, auch wo wir uns schon auf phonologischer terra firma befinden. Untersuchungen der vokalischen (V-V) Assimilation UND der Rolle von Konsonanten in dieser Assimilation passen beide zu den germanischen Umlautfakten.

\section{Schlußfolgerungen}

Vorteile vom "ingeneraten" Umlaut gegenüber der Twaddellschen Umlauttheorie:

Vermeidet deskriptive Wiedersprüche der alten Theorie(n).

Erfaßt wesentlich mehr Daten:

- Hinderung von Primärumlaut (paßt sogar zur ahd Dialekt-phonologie/-phonetik)

- Oberdeutsche Umlautfeindlichkeit (also moderne Dialektphonologie)

- niederländische Dialektgeographie (also westgermanische Dialektgeographie)

Erfaßt die natürliche evolutionäre Bahn von Umlaut:

- Phonetische Basis von allen Umlauterscheinungen in der Koartikulation

- Allmähliche Entfaltung vom komplexen Lautwandel (à la Ohala, u.a.)

- Wichtige direkte Parallelen in den modernen Tochtersprachen

Paßt nahtlos in moderne Theorien der Phonologie und des Lautwandels. 


\section{LITERATUR}

Antonsen, Elmer. 1969. Zur Umlautfeindlichkeit des Oberdeutschen. Zeitschrift für Dialektologie \& Linguistik 36.201-07.

Bladon, A. \& A. Al-Bamerni. 1976. 'Coarticulation resistance in English //.' J. of Phonetics 4.137-150.

Braune, W. \& H. Eggers. 1987. Althochdeutsche Grammatik. 14th ed. Tübingen: Niemeyer.

Britain, David. 1997. 'Dialect contact and phonological reallocation: "Canadian Raising" in the English Fens.' Language in Society 26.15-46.

Buccini, Anthony F. 1992. The development of umlaut and the dialectal position of Dutch in Germanic. PhD dissertation: Cornell University.

Butcher, Andrew \& Eckart Weiher. 1976. 'An electropalatographic investigation of coarticulation in VCV sequences.' Journal of Phonetics 4.59-74.

Cohn, Abigail \& Ayako Tsuchida. 1999. Sonorant devoicing and the phonetic realization of [spread glottis] in English." Paper presented at the Linguistic Society of America. Los Angeles.

Davis, Garry W. \& Gregory K. Iverson. 1995. 'Segment Organization in the High German Consonant Shift.' American Journal of Germanic Linguistics 7(2).111-127.

Elgersma, Diana \& Paul Houseman. 1997. 'Morphological OT: The use of umlaut in Modern Standard German plural marking.' Paper presented at GLAC 3, UCLA.

Farnetani, Eddia. 1997. Coarticuiation and connected speecn prucessos. ihe fianaiovok of Phonetic Sciences, ed. by William J. Hardcastle and John Laver, pp. 371-404. Cambridge, Mass.: Blackwell.

Fertig, David. 1996. 'Phonology, orthography and the umlaut puzzle', Diachronic \& syntactic studies in Germanic Linguistics, ed. R. Lippi-Green \& J. C. Salmons, pp. 169-184. Amsterdam: Benjamins.

Holsinger David J. \& Joseph C. Salmons. Toward "a complete analysis of the residues": On regular vs. morpholexical approaches to Old High German umlaut. Festschrift for E.F. Konrad Koerner, ed. by Sheila Embleton, et al. Amsterdam: Benjamins.

Howell, Robert B. 1988. 'Proto-Germanic */X/ and Gothic Breaking." Semper idem et novus, ed. F. Gentry, pp. 27-55. (Göppinger Arbeiten zur Germanistik, 481.) Göppingen: Kümmerle. 1991 Old English breaking and its Germanic analogues. Tübingen: Max Niemeyer. \& Joseph C. Salmons. 1997. 'Umlautless residues in Germanic.' American Journal of Germanic Linguistics 9(1).83-111.

Iverson, Gregory K., Garry W. Davis \& Joseph C. Salmons. 1994. 'Umlaut blocking environments in Old High German.' Folia Linguistica Historica 15.131-48.

\& Joseph C. Salmons. 1996. 'The primacy of primary umlaut.' $P B B$ 118.69-86. $\&$. 1999. Umlaut as Regular Sound Change: The phonetic basis of "ingenerate umlaut". Festschrift for W. P. Lehmann, ed. by E.C. Polomé \& Carol Justus. 154-71.

Keating, Patricia A. 1988. 'Underspecification in Phonetics.' Phonology 5.275-292.

King, Robert D. 1969. Historical linguistics and generative grammar. Englewood Cliffs: Prentice-Hall.

Kiparsky, Paul . 1985. 'Some consequences of Lexical Phonology.' Phonology Yearbook 2.85138.

Krygier, Marcin. 1997. From Regularity to Anomaly: Inflectional i-Umlaut im Middle English. Frankfurt: Peter Lang.

Labov, William. 1972. Sociolinguistic Patterns. Philadelphia: University of Pennsylvania Press.

Magen, H. S. 1989. 'An acoustic study of vowel-tovowel coarticulation in English.' PhD diss.: Yale U.

Manuel, S. 1987. Acoustical and perceptual consequences of vowel-to-vowel coarticulation in three Bantu languages. PhD dissertation: Yale University. 
1990. 'The role of contrast in limiting vowel-to-vowel coarticulation in different languages.' Journal of the Acoustical Society of America 88.1286-1298.

Noreen, A. 1970. Altnordische Grammatik. University: Univ. of Alabama Press/Tübingen: Niemeyer.

Ohala, John J. 1993. 'Coarticulation and Phonology.' Language \& Speech 36.155-70.

Öhman, S. 1966. 'Coarticulation in VCV utterances: Spectrographic measurements.' Journal of the Acoustical Society of America 39.151-268.

Paul, H., P. Wiehl \& S. Grosse. 1989. Mittelhochdeutsche Grammatik. Tübingen: Max Niemeyer. 23. ed.

Penzl, Herbert. 1949. 'Umlaut and secondary umlaut in Old High German.' Language 25.223-240.

. 1994. 'Historiographie und Sprachgeschichte: Zur Beschreibung des althochdeutschen iUmlauts.' American Joumal of Germanic Linguistics \& Literatures 6.51-62.

Recasens, D. 1984. 'V-to-C coarticulation in Catalan VCV sequences: An articulatory and acoustical study.' Journal of the Acoustical Society of America 91.2911-2925.

Robinson, Orrin W. 1975. 'Abstract phonology and the history of umlaut.' Lingua 37.1-29.

Russ, Charles V. J. 1977. 'Die Entwicklung des Umlauts im Deutschen im Spiegel verschiedener linguistischer Theorien.' $P B B$ 99.213-241.

Schirmunski, Viktor M. 1962. Deutsche Mundartkunde. Berlin: Akademie Verlag.

Schulte, Michael. 1998. Grundfragen der Umlautphonemisierung. Berlin: de Gruyter.

Smith, Regina. Forthcoming. 'Testing compensatory theories of umlaut.' Leuvense Bijdragen.

Twaddell, W. Freeman. 1938. 'A note on OHG umlaut.' Monatshefte 30.177-181.

Wiese, Richard. 1996. The Phonology of German. Oxford: Clarendon Press. 